\title{
Acoustic calibration of the Exterior Effects Room at the NASA Langley Research Center
}

\author{
Kenneth J. Faller II ${ }^{\mathrm{a}}$, Stephen A. Rizzi ${ }^{\mathrm{b}}$, Jacob Klos ${ }^{\mathrm{b}}$, William L. Chapin ${ }^{\mathrm{c}}$, \\ Fahri Surucu' ${ }^{\mathrm{c}}$, and Aric R. Aumann ${ }^{\mathrm{d}}$ \\ ${ }^{a}$ NASA Postdoctoral Program, Structural Acoustics Branch, NASA Langley Research Center \\ Hampton, VA 23681-2199, USA \\ ${ }^{b}$ Structural Acoustics Branch, NASA Langley Research Center, Hampton, VA 23681-2199, USA \\ ${ }^{c}$ AuSIM, Inc., Mountain View, CA 94043-4514, USA \\ ${ }^{d}$ Analytical Services and Materials, Inc., Hampton, VA 23666, USA
}

\begin{abstract}
The Exterior Effects Room (EER) at the NASA Langley Research Center is a 39-seat auditorium built for psychoacoustic studies of aircraft community noise. The original reproduction system employed monaural playback and hence lacked sound localization capability. In an effort to more closely recreate field test conditions, a significant upgrade was undertaken to allow simulation of a three-dimensional audio and visual environment. The 3D audio system consists of 27 mid and high frequency satellite speakers and 4 subwoofers, driven by a real-time audio server running an implementation of Vector Base Amplitude Panning. The audio server is part of a larger simulation system, which controls the audio and visual presentation of recorded and synthesized aircraft flyovers. The focus of this work is on the calibration of the 3D audio system, including gains used in the amplitude panning algorithm, speaker equalization, and absolute gain control. Because the speakers are installed in an irregularly shaped room, the speaker equalization includes time delay and gain compensation due to different mounting distances from the focal point, filtering for color compensation due to different installations (half space, corner, baffled/unbaffled), and cross-over filtering.
\end{abstract}

PACS: $43.55 . J z$ 


\section{INTRODUCTION}

\section{Objective and Approach}

- Objective-Calibrate a new three-dimensional audio system in the NASA Langley Exterior Effects Room (EER) for use in subjective testing of aircraft community noise.

- The audio system is a real-time, multi-channel, loudspeaker-based system utilizing amplitude panning for placement of virtual sources

- Approach - Develop panning gains using loudspeaker position data and loudspeaker equalization using measured response data.

Figure 1

The objective of this research is to calibrate the three-dimensional (3D) audio system, in the NASA Langley Exterior Effects Room (EER), for use in subjective testing of aircraft community noise. The audio system is a realtime, multi-channel, speaker-based system that utilizes Vector-Base Amplitude Panning, or VBAP (see [1-2]), for placement of virtual sources. The VBAP gains were developed using the speaker position data. Speaker equalization filters were generated and analyzed using measured response data.

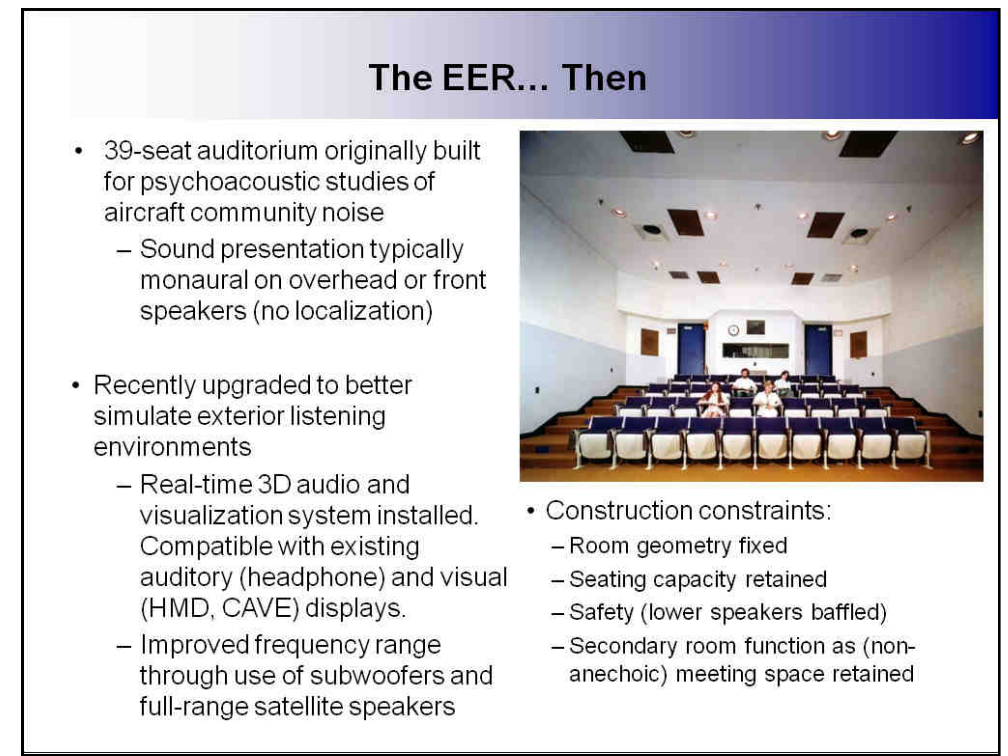

Figure 2

The EER is a 39-seat auditorium originally built to study the psychoacoustic effects of aircraft community noise. The original reproduction system employed monaural playback and hence lacked sound localization capability. Recently, the EER was upgraded to better simulate exterior listening environments. The upgrades included a realtime 3D audio and visual system which is compatible with previously existing auditory (headphone) and visual (Head-Mounted Display, CAVE ${ }^{\mathrm{TM}}$ ) displays. Improved frequency range was also achieved through the installation 
of subwoofers and full-range satellite speakers. However, several construction constraints were required which affected the overall spectral colorization of the EER. These constraints were: fixed room geometry, retention of prior seating capacity and baffling of satellite speakers. Baffling of the satellite speakers was required to protect equipment and personnel as the room also functions as a non-anechoic meeting space.

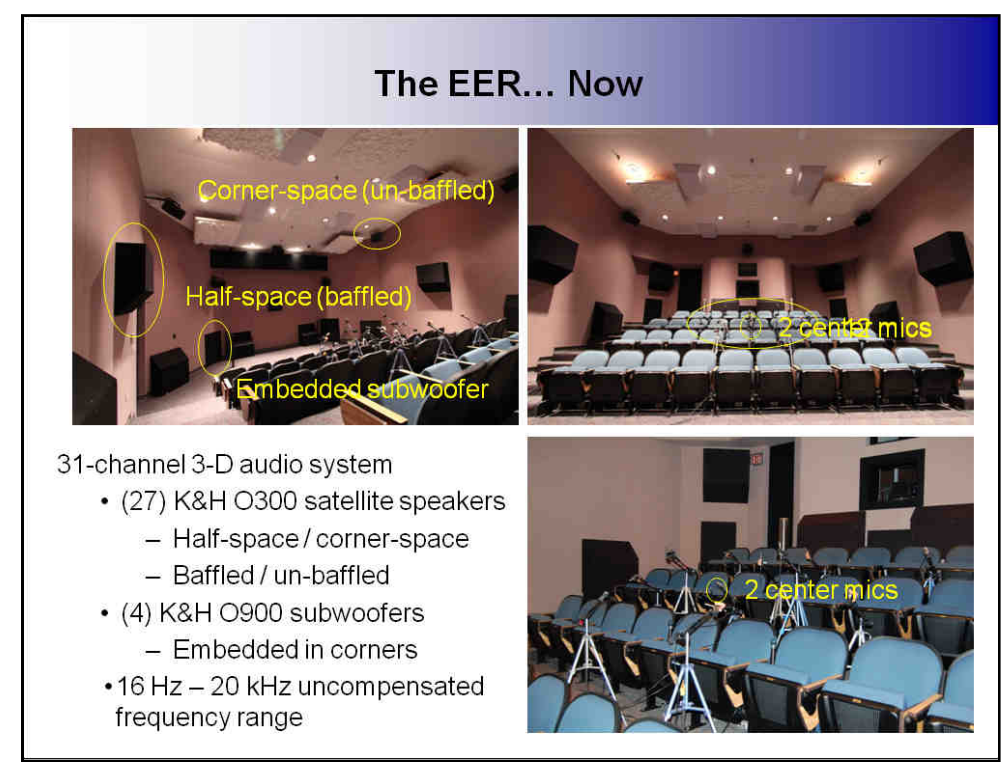

Figure 3

The EER now is equipped with a 31-channel 3D audio system comprised of twenty-seven K\&H O300 satellite speakers and four K\&H O900 subwoofers. Together these speakers provide a $16 \mathrm{~Hz}$ to $20 \mathrm{kHz}$ uncompensated frequency range. The satellites are located in various locations throughout the EER and the subwoofers are embedded in the corners of the room. As indicated above, the satellite speakers located in the lower half of the room are baffled to protect both the speakers and test subjects. Also shown in Figure 3 on the right, is the microphone setup used to obtain the measurement data needed to generate the speaker equalization filters. A centrally located grouping of two microphones, and a broader array of twelve microphones were used for speaker equalization. 


\section{3D SPATIAL SOUND PROCESSING}

The following subsections will describe the techniques employed for the $3 \mathrm{D}$ spatial sound processing utilized in the EER.

\section{A. Vector-Base Amplitude Panning (VBAP)}

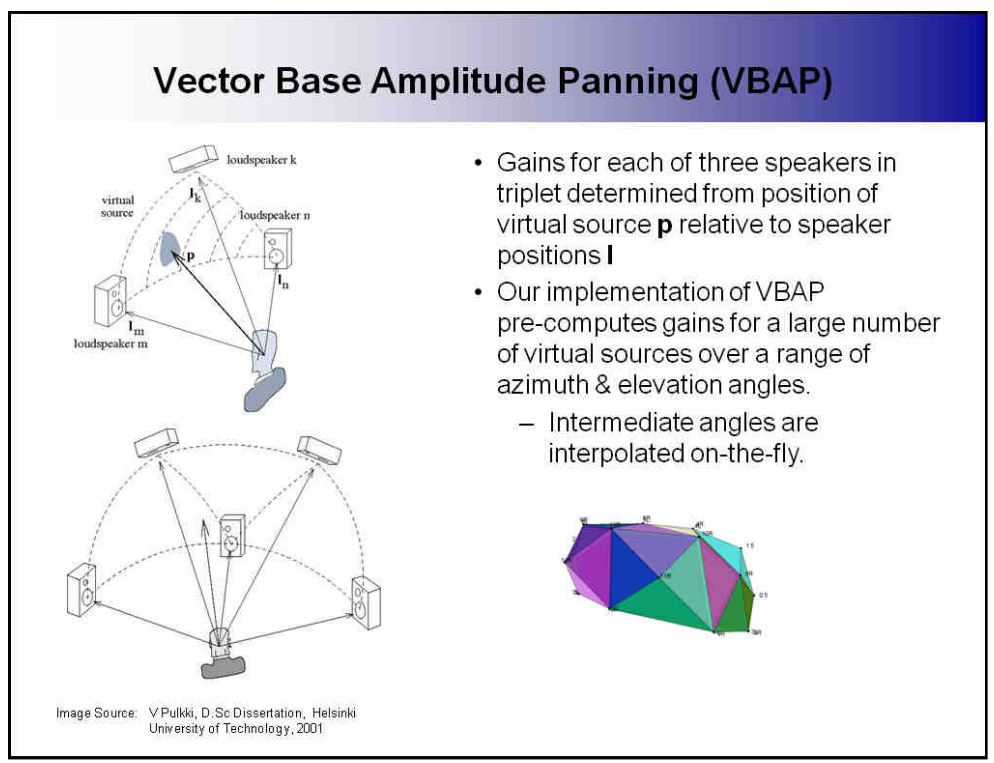

Figure 4

For conventional stereo panning, two speakers are used and a virtual source is panned, to the left or right, in front of a listener. Vector-Base Amplitude Panning (VBAP) is an extension of this, however, a speaker triplet is used [12]. From the view of the listener, the speaker triplet forms a triangle and, depending on the ratio of the gains of each speaker, the listener will perceive a virtual source inside this triangle. The gains, $\mathbf{g}$, of each of the speakers in the triplet are determined from the position of the virtual source, $\mathbf{p}$, relative to the speaker position, l:

$$
\begin{aligned}
& \mathbf{p}=g_{n} \mathbf{l}_{n}+g_{m} \mathbf{l}_{m}+g_{k} \mathbf{l}_{k} \\
& \mathbf{g}=\mathbf{p}^{T} \mathbf{L}_{n m k}^{-1}
\end{aligned}
$$

where $\mathrm{L}_{n m k}=\left[\mathrm{l}_{n} \mathbf{l}_{m} \mathbf{l}_{k}\right]^{T}$. The gains are pre-computed for a finite number of virtual sources at regularly spaced grid points within the triangle and intermediate locations are estimated using interpolation. It is desired to represent an immersive exterior listening environment with the EER which requires the ability to pan a virtual source completely around the listener. This is achieved by surrounding the listener with speakers and forming non-overlapping triangles between them. The gains can then be computed for a large number of grid points, over a range of azimuth and elevation angles. Interpolation is used for the intermediate angles. 


\section{B. Triangulation and Spread Angle}

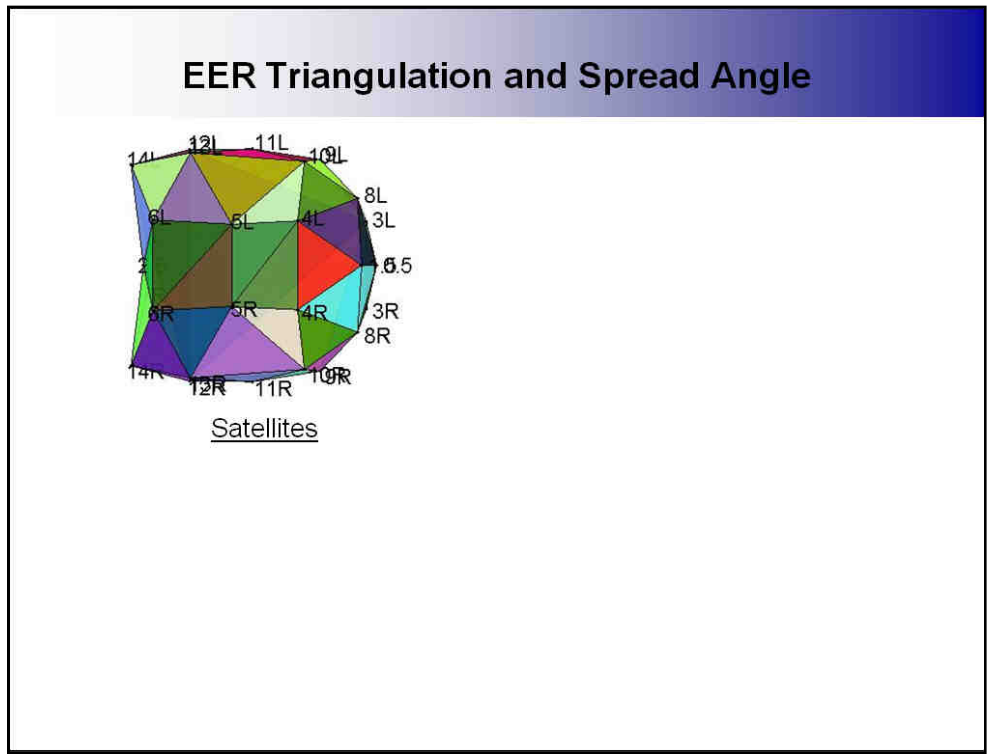

Figure 5

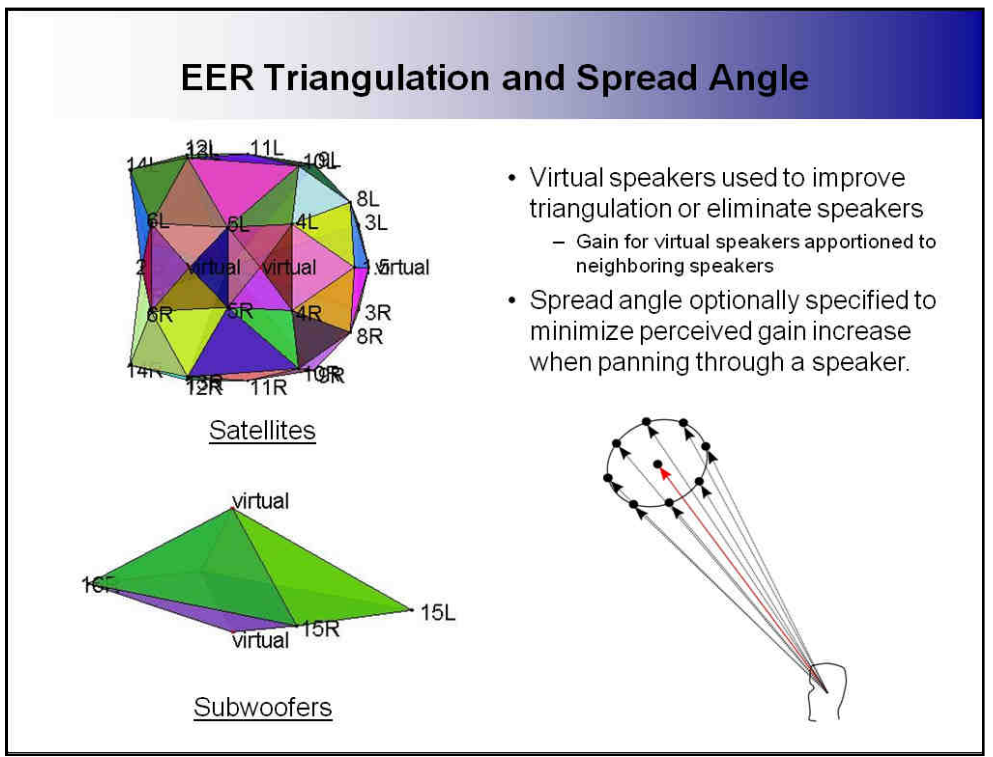

Figure 6

Certain speaker configurations in the EER result in less than ideal triangulations. For example, consider the speakers on the ceiling which are positioned in a rectangular formation. Triangulation, for this instance, is formed by connecting a pair of the diagonal vertices of the rectangle (Figure 5). However, a virtual source is perceived to oscillate as it is panned through this resulting triangulation. This is attributed to the abrupt change in excited speakers as the virtual source moves from one triangle to the next. An improvement in perception is achieved if a virtual speaker is positioned at the center of the rectangle and the gain factor of it is apportioned to the neighboring speakers (Figure 6, top left). This can also be used to eliminate speakers which may be affected by objects within the EER. For example, the speaker directly in front of the EER is obstructed when the projection screen is down. This results in a noticeable decrease in volume when a virtual source is panned through the corresponding triangles.

Another improvement of the 3D spatial sound processing is spread angle. Spread angle can be optionally specified to minimize perceived gain increases when panning a virtual source through a speaker. This results from 
the focusing of all the gain energy on a single speaker, which can be avoided by defining a spread of additional virtual sources around the current virtual source. The gains of the additional virtual sources are averaged and used as the gain for the current virtual source.

\section{SPEAKER EQUALIZATION}

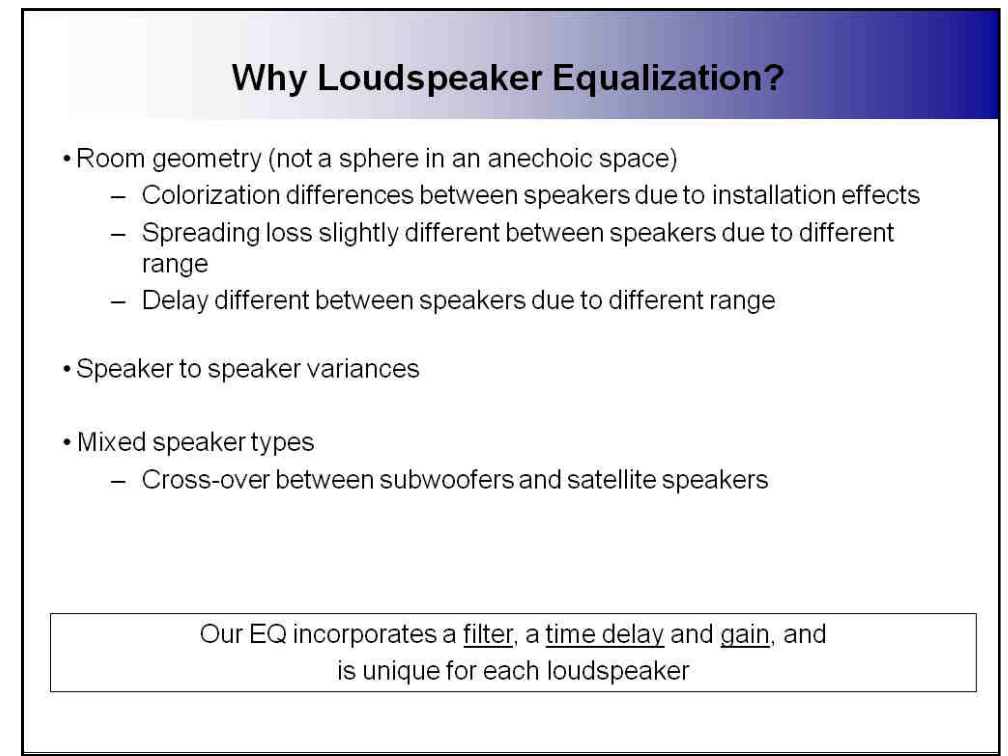

Figure 7

There are several reasons why speaker equalization is necessary for the EER. The EER is not a perfect sphere in an anechoic space. As a result, the speakers have spectral colorization differences, due to installation effects, and range differences which affect the delay and spreading loss of each. Additional colorization is attributed to the variation and cross-over differences between speakers. The next subsection summarizes the speaker equalization filter used to compensate for all of these effects, which incorporates a filter, a time-delay, and gain unique to each speaker.

\section{A. Speaker Equalization Filters}

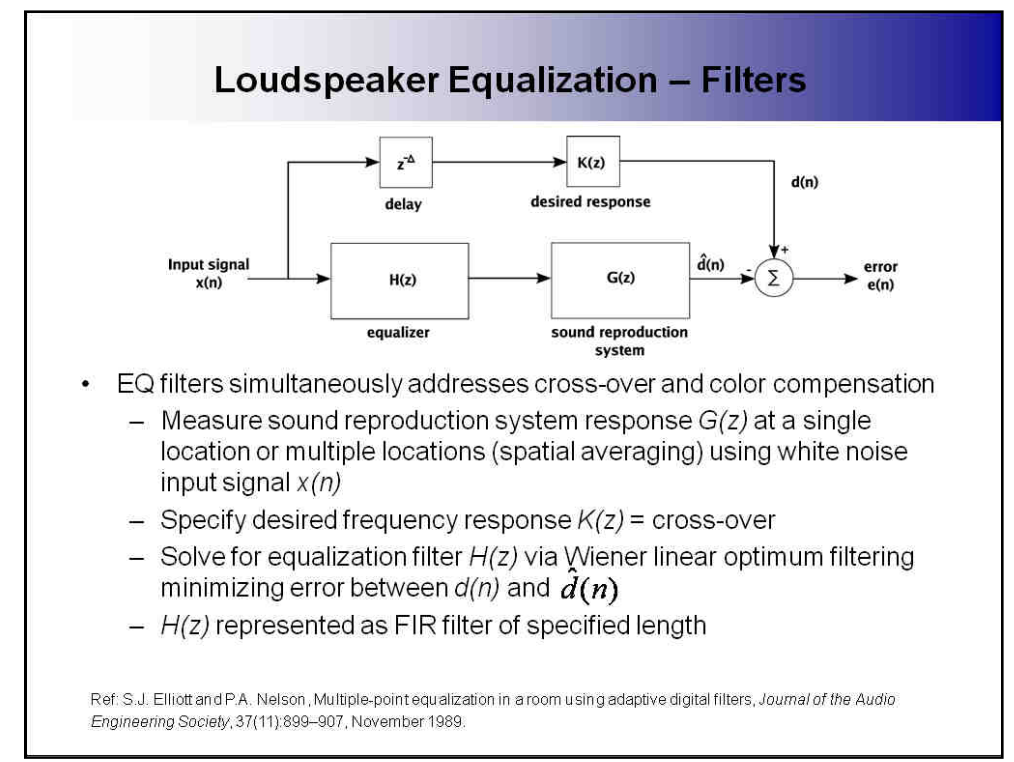

Figure 8 
The speaker equalization filters simultaneously address cross-over and color compensations. The first step in the process of developing speaker equalization filters is to measure the sound reproduction system response, $G(z)$, at a single location or multiple locations (spatial averaging) using a white noise input signal, $x(n)$. The desired frequency response, $k(z)$, is then specified. For the EER, $k(z)$ was specified to be the desired cross-over for the corresponding speaker. Finally, the equalization filter, $H(z)$, is computed using the Wiener linear optimum filtering approach which minimizes the error between the desired response, $d(n)$, and the compensated system response, $\hat{d}(n)[3] . H(z)$ is represented as a FIR filter of specified length.

\section{RESULTS}

\section{A. Subwoofer Equalization}

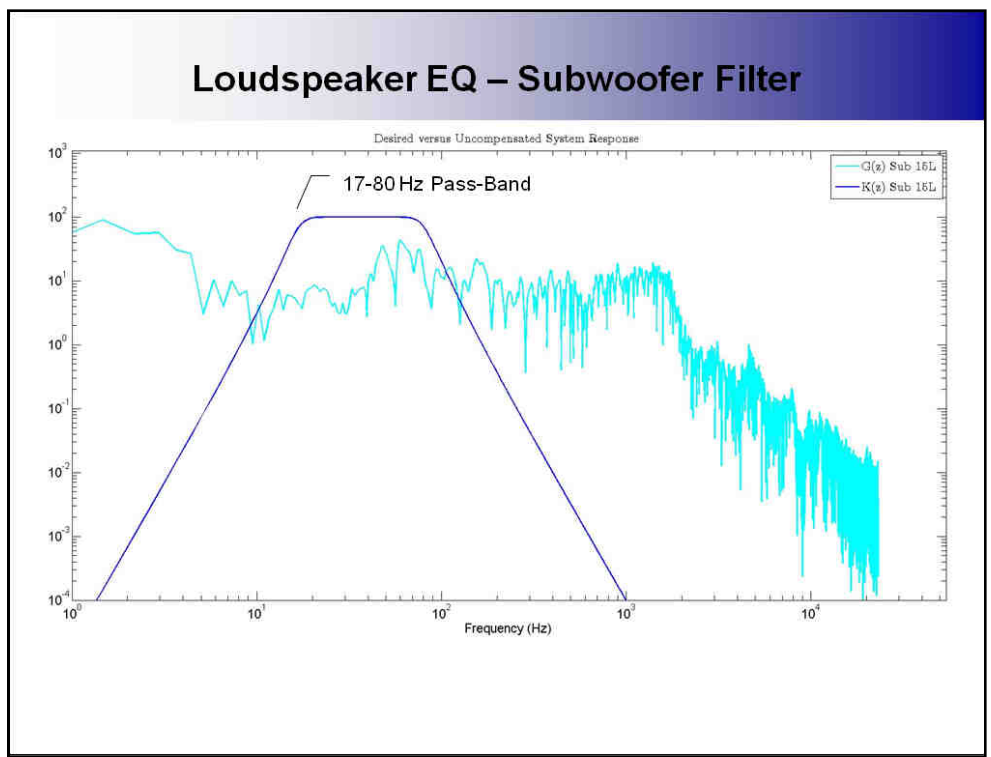

Figure 9

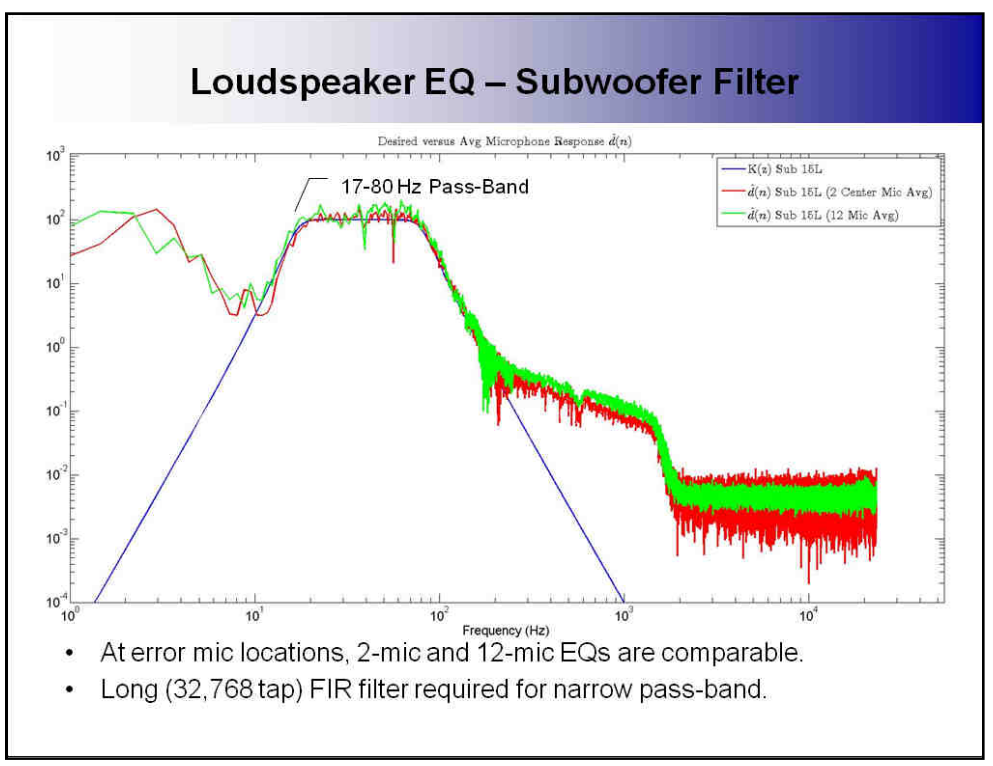

Figure 10 
Figure 9 shows the raw system response, $G(z)$, for subwoofer $15 \mathrm{~L}$ in the EER (light blue) and the desired response $K(z)$ (blue). The pass-band of the desired response $K(z)$ was selected to be between 17 to $80 \mathrm{~Hz}$ because $17 \mathrm{~Hz}$ is the lower limit of the subwoofers and $80 \mathrm{~Hz}$ was selected to minimize any spatialization contribution from the subwoofers. Figure 10 shows the compensated system responses, $\hat{d}(n)$, when they are measured at a narrow $(2$ center microphone) sweet spot (red) and broad (12 microphone) sweet spot (green). The compensated system responses were comparable at the error microphone locations. To achieve this equalization performance for this narrow of a pass-band, long (32k tap) FIR filters were required. However, processing multiple filters of this size exceeds the real-time computational capabilities of the audio server in the EER. Therefore, methods of generating reduced length IIR surrogates need to be explored.

\section{B. Satellite Equalization}

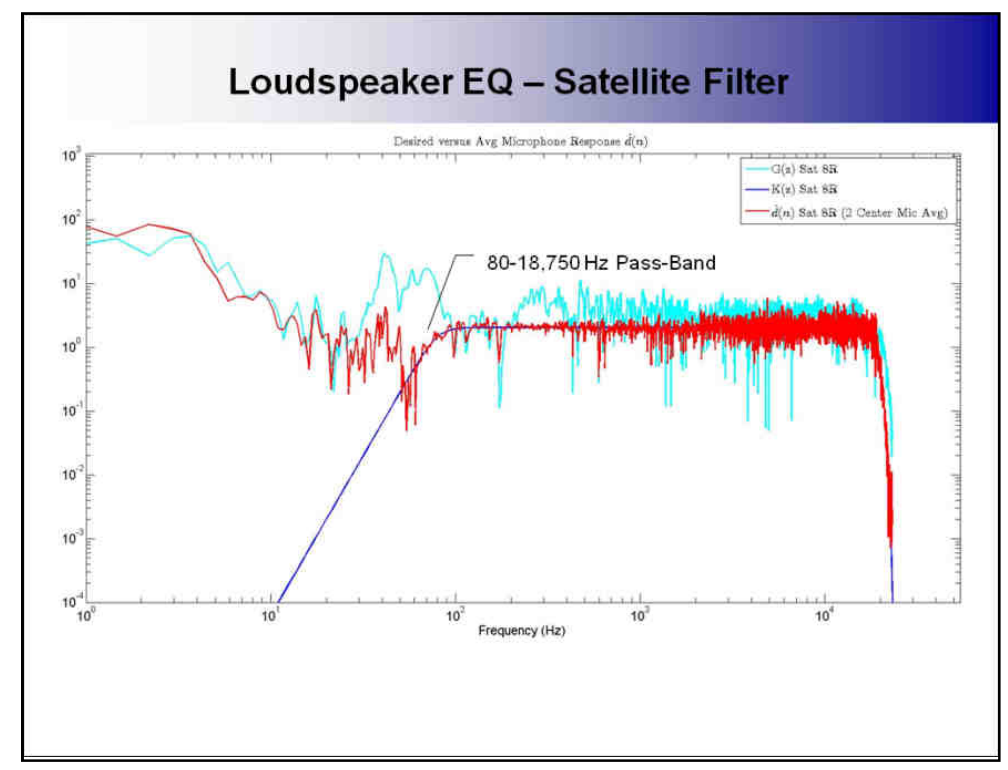

Figure 11

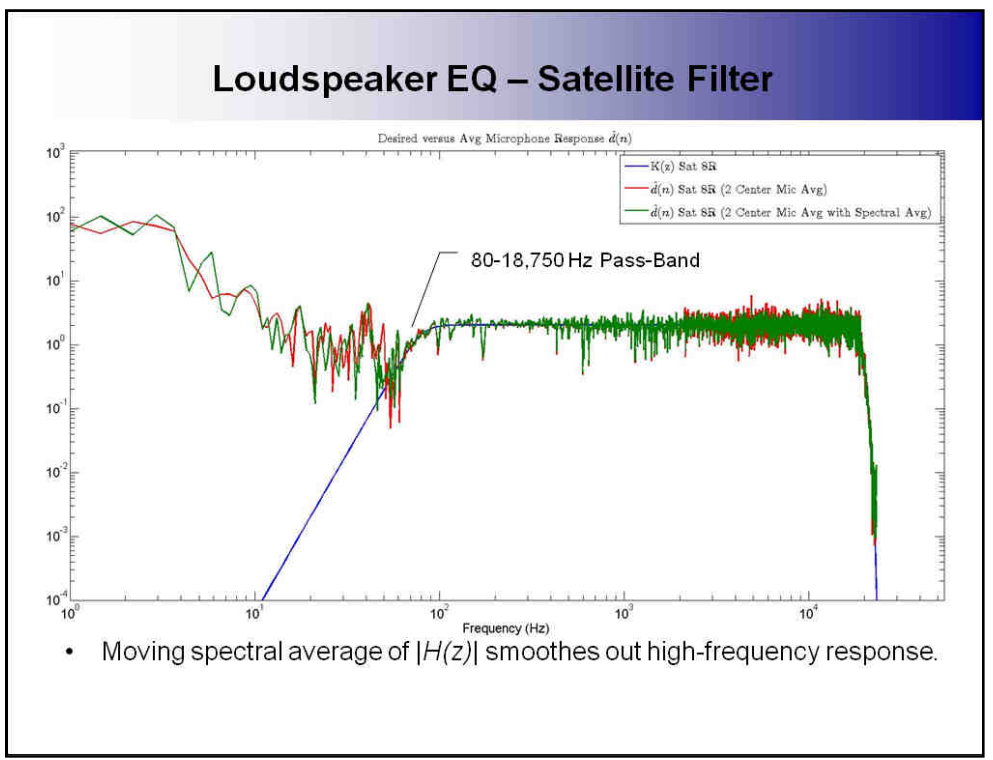

Figure 12 


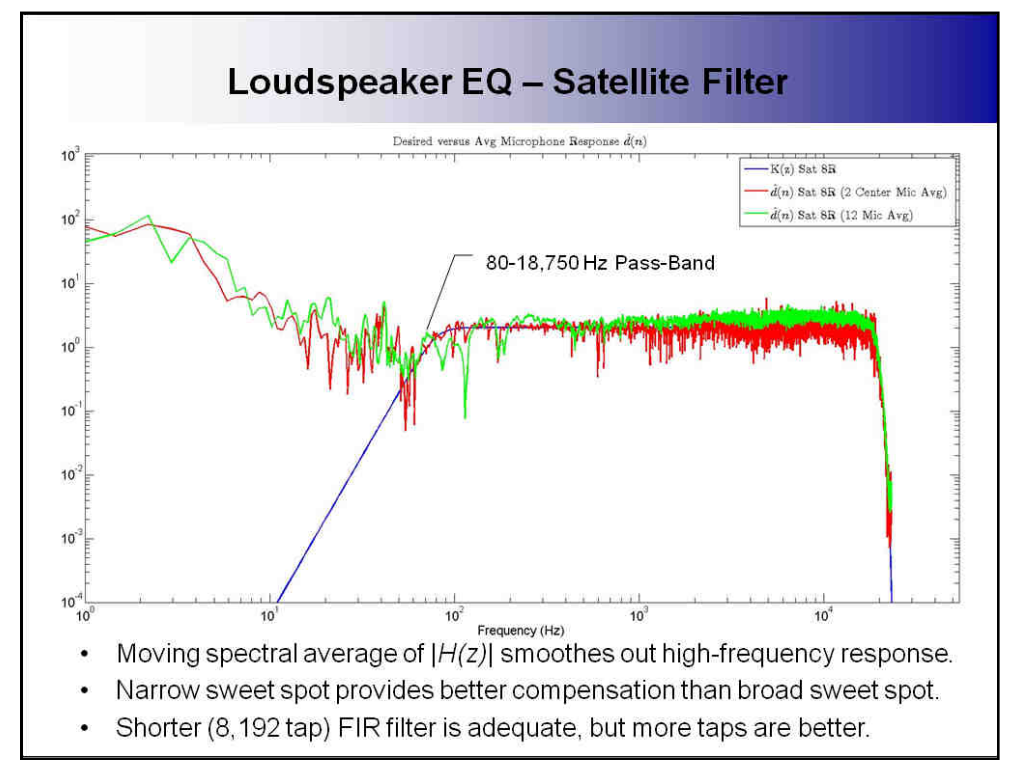

Figure 13

Figure 11 shows the raw system response, $G(z)$, for satellite $8 \mathrm{R}$ in the EER (light blue), the desired response $K(z)$ (blue), and the compensated system response, $\hat{d}(n)$, when it is measured at a narrow sweet spot (red). As can be seen, the compensated system response, $\hat{d}(n)$, is improved by the equalization filter in comparison to the raw system response, $G(z)$. Further improvement is achieved, for the high frequencies, when the equalization filter is spectrally averaged prior to obtaining the compensated system response, $\hat{d}(n)$ (Figure 12). The equalization filters created based on the narrow sweet spot resulted in better compensation than those based on the broad sweet spot (Figure 13). Adequate equalization was achieved with shorter ( $8 \mathrm{k}$ tap) FIR filters for the satellite speakers. However, longer FIR filters result in superior equalization.

\section{Time Delay}

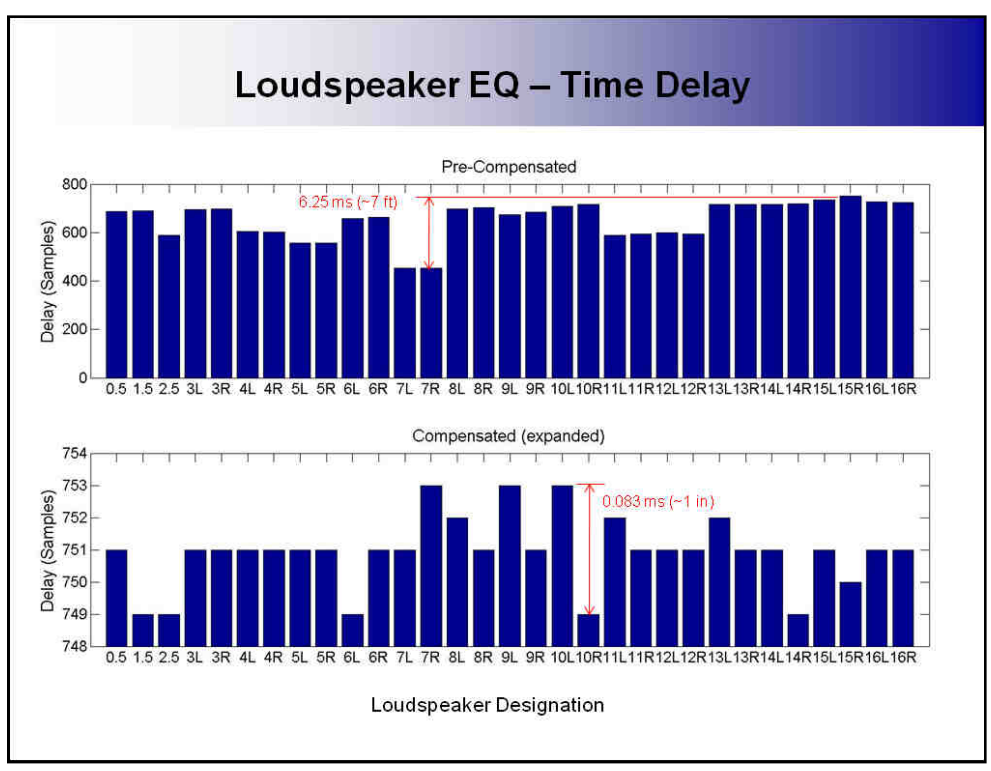

Figure 14

The time delays were estimated from the speaker Impulse Responses (IRs) obtained using Golay codes [4]. Figure 14 shows the uncompensated (top) and compensated (bottom) time delays for each speaker in the EER. As 
can be seen, there is a maximum difference of about 300 samples (about $6.25 \mathrm{~ms}$ at a sampling rate of $48 \mathrm{kHz}$ or about 7 feet) between the smallest and largest time delays. After compensation, the delay difference is reduced to four samples $(0.083 \mathrm{~ms}$ at a sampling rate of $48 \mathrm{kHz}$ or about $1 \mathrm{inch})$.

\section{CONCLUSION AND FUTURE WORK}

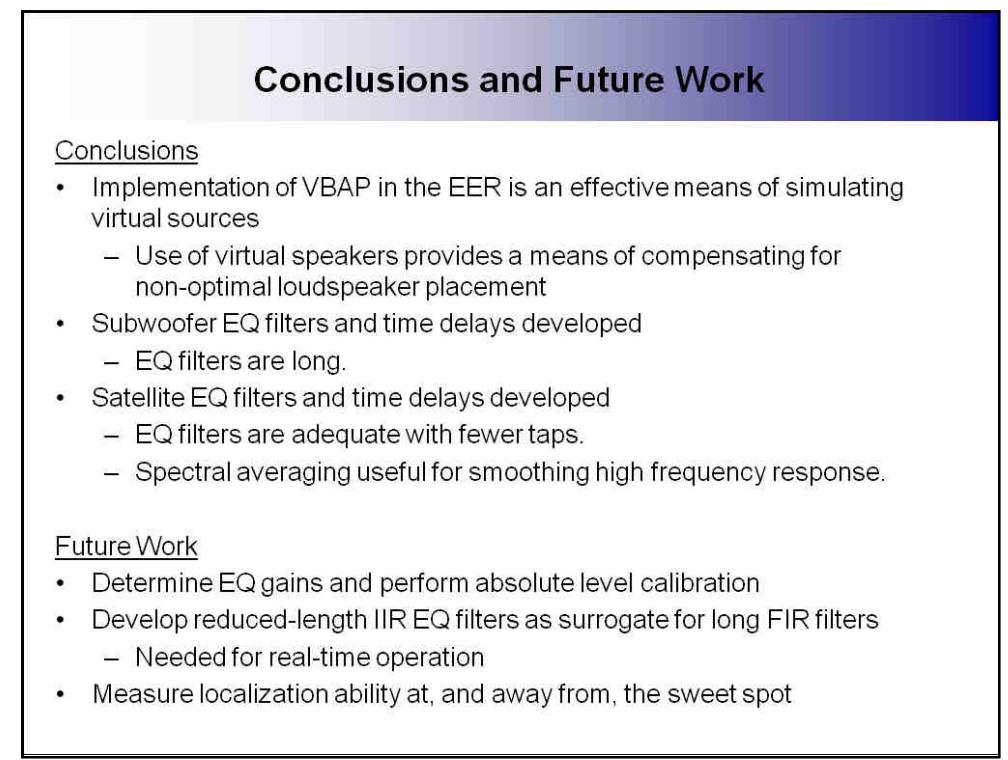

Figure 15

In conclusion, the implementation of VBAP used in the EER is an effective means of simulating sound sources in three-dimensional space. Additionally, virtual speakers provided a means to eliminate non-ideal speakers and to improve triangulations. Creation of speaker equalization filters for the subwoofer and satellite speakers was successful. The equalization filters for the satellite speakers required fewer taps than subwoofer equalization filters to achieve adequate equalization. Furthermore, the use of spectral averaging improved the high frequency response of the satellite speakers. However, the computational needs to process the long subwoofer equalization filters in real-time is beyond the capabilities of the audio server in the EER. Hence, reduce-length IIR surrogates for the long FIR filters need to be explored as a potential solution. Some other future work will include determining and compensating for the absolute gain of the speakers after equalization measurement of the localization ability at and away from the central sweet spot.

\section{ACKNOWLEDGMENTS}

This research was supported by an appointment to the NASA Postdoctoral Program at the NASA Langley Research Center (LaRC), administered by Oak Ridge Associated Universities (ORAU) through a contract with NASA.

\section{VII.REFERENCES}

1. V. Pulkki, Spatial Sound Generation and Perception by Amplitude Panning Techniques. 2001, Helsinki University of Technology: Espoo, Finland. p. 42.

2. V. Pulkki, Virtual Sound Source Positioning Using Vector-Base Amplitude Panning. Journal of the Audio Engineering Society, 1997. 45(6): p. 456-466.

3. S. Elliott and P. Nelson, Multiple-Point Equalization in a Room Using Adaptive Digital Filters. Journal of the Audio Engineering Society, 1989. 37(11): p. 899-907.

4. S. Foster, Impulse Response Measurement Using Golay Codes, in IEEE International Conference on Acoustics, Speech, and Signal Processing (ICASSP). 1986: Tokyo, Japan. p. 929-932. 